Results: DDC feeding led to increased serum markers of hepatic injury, ductular reaction, induction of proinflammatory cytokines and biliary fibrosis. Interestingly, melittin treatment attenuated hepatic function marker (ALT, AP, total bilirubin), ductular reaction (Cytokeratin 19), reactive phenotype of cholangiocytes and cholangitis (VCAM and CDb11) and biliary fibrosis (type I collagen, TIMP-1, TGF-b).

Conclusion: Our data suggest that melittin treatment can be protective for chronic cholestasis disease as well in DDC feeding mice. Further study for the anti-inflammatory capacity of melittin is given target therapy in cholangiopathy.

\section{CORRELATION VEGF AND MVD WITH RESPONSE TO RADIOTHERAPY IN ADENOCARCINOMA CERVICAL}

\section{Sri Suryanti, Bethy S. Hernowo, Fennisia Wibisono}

Department of Anatomical Pathology Faculty of Medicine University Padjajaran/General Hospital Dr. Hasan Sadikin, Bandung, Indonesia

Introduction: Radiation therapy plays a critical role in women with advanced-stage cervical cancer worldwide including adenocarcinoma type, particularly in developing countries, and most of the time it may be the only available treatment. The efficacy of radiation largely depends on the radiosensitivity of the tumor. Neovascularization is an important step in the tumor progression and the therapeutic targeting of the tumor blood vessels appears to be a good strategy to follow in the anti-cancer treatment. Thus, even in an incipient phase of the clinical research process, the combination between the anti-angiogenic aimed therapies and the current radio-chemotherapy seems to represent a new, feasible and promising approach. The aim of the present study was to determine the prognostic and/or predictive value of some biological markers of tumor angiogenesis and of their implication in increasing the efficacy of current treatments for this cancer.

Methods: So far, 32 women were included in a retrospective trial. A tumor biopsy was obtained from each patient before the start of therapy and patients with histopathological report as adenocarcinoma cervical were included in this study. The density of microvascularization was assessed using CD34 monoclonal antibody, the expression of angiogenic factors VEGF were determined in tumor biopsies by specific immunohistochemistry techniques, using primary antibodies anti-VEGF respectively.

Results: Among the studied clinical and molecular factors, we found to be predictive for the type of response the following factors was MVD expression. MVD expression has higher correlation with response to radiotherapy than VEGF expression.

Conclusions: MVD has significant value in predicting response to radiotherapy in adenocarcinoma cervical cancer patients than VEGF expression.

\section{MULTINODULAR GOITER WITH ADIPOSE METAPLASIA: A CASE REPORT}

Worapop Suthiwartnarueput

Department of Pathology and Forensic Medicine, Faculty of Medicine, Thammasat University, Thailand
Background: The presence of adipose tissue within the thyroid gland has been reported in wide range of conditions such as adenolipoma (thyrolipoma), thyrolipomatosis, amyloid goiter, goiter, follicular adenoma, papillary carcinoma, lymphocytic thyroiditis and heterotopic nest. Several theories have been proposed such as metaplastic change from stromal fibroblasts due to local hypoxia or developmental abnormality during embryogenesis.

Case report: The author reports a case of an 82-year-old woman who presented with a 60-year history of midline neck mass with gradual onset of compressive symptom for the past several months. Computed tomography of the neck showed diffuse thyroid enlargement with features suggestive of multinodular goiter extending to the superior mediastinum. Total thyroidectomy was performed. Gross examination revealed a huge thyroid gland with nodular surface measuring $14 \times 9.8 \times 5 \mathrm{~cm}$. and weighing $320.5 \mathrm{~g}$. Serial sections showed multiple colloidal brown nodules of $1-6.2 \mathrm{~cm}$. in size, with thin fibrous capsule and degenerative change involving the bilateral lobes and isthmus. Histologic sections displayed benign follicular proliferation of various sizes with degenerative change and scattered infiltration by mature adipose tissue in all nodules. The final diagnosis was multinodular goiter with mature adipose tissue infiltration, most likely from adipose metaplasia due to prolonged degenerative change.

Conclusion: Thyroid infiltration by mature adipose tissue has been described in various conditions. The pathogenesis is unclear. The diagnosis requires combinations of clinical details, gross pathology and histopathology.

\section{CLINICOPATHOLOGICAL FINDINGS IN A CASE SERIES OF EXTRAPLEURAL SOLITARY FIBROUS TUMOURS}

\author{
Edwin $\operatorname{Tan}^{1,2}$, Rohan Lourie ${ }^{2}$ \\ ${ }^{1}$ Royal Brisbane and Women's Hospital, and ${ }^{2}$ Mater Pathology, \\ Queensland, Australia
}

Background: Extrapleural solitary fibrous tumours (SFTs) represent an uncommon soft tissue tumour with unpredictable behavior.

Aims: This case series illustrates the pathological features that maybe associated with recurrences.

Methods: The pathology and clinical reports were retrieved from the electronic databases from two institutions between 2001 and 2014 and included thirteen $(n=13)$ extrapleural SFTs patients in our analysis. The patients were followed up between 1 month to 13 years and 8 months (mean: 3 years and 1 month).

Results: There were 10 males and 3 females, whose age ranged from 28 to 79 years (mean: 51.5 years). The documented tumours size ranged from 20 to $125 \mathrm{~mm}$ (mean: $61.8 \mathrm{~mm}$ ). Seven tumours were located in the abdomen or pelvis, two in the extremity and four, head and neck. Twelve tumours had circumscribed borders and one had infiltrative borders. Eleven tumours were clear of the resection margins and two were incompletely resected. The risk of recurrence was stratified based on the patient's age, tumour size and number of mitotic figures. Two tumours had features associated with high risk of recurrence, two had moderate risk and nine had low risk. Two of the tumours were recurrences where the initial histology had low risk features. One of these tumours had low risk features, was incompletely excised and was suspicious for a further recurrence. No 
recurrences were detected among the remaining patients during the follow up period.

Conclusions: Our series suggest that recurrence can occur in extrapleural SFTs even with low risk features. The treatment of choice is complete resection followed by extended follow-up surveillance.

\section{MUSCLE PATHOLOGY: TEACHING OPPORTUNITIES AND STATEGIES IN A PATHOLOGY DEPARTMENT IN SINGAPORE}

Kong-Bing Tan, Yoke-Sun Lee

Department of Pathology, Yong Loo Lin School of Medicine, National University Health System, Singapore

Background: Muscle pathology is a highly sub-specialized discipline in histopathology. While it does not generally feature prominently in routine histopathology practice or in fellowship or board examinations, some training exposure to the discipline for histopathology trainees is often deemed desirable.

In Singapore, histopathology trainees are given exposure to the discipline through sessions in the national training programme, intradepartmental neuropathology teaching as well as opportunistically during work at pathology departments.

Implementation: During recent national training programme teaching sessions, muscle pathology was introduced via a lecture on the general approach to muscle biopsy interpretation and a separate presentation of short clinico-pathological cases with quizzes. Conditions covered include muscular dystrophy, mitochondrial myopathy, central core myopathy, glycogen storage disease, polymyositis, disuse atrophy and neurogenic atrophy. In our department, trainees undertake structured training in the major subspecialties of histopathology, including neuropathology. They receive weekly illustrative slides for preview, culminating in a multi-header teaching session with the teaching pathologist. For neuropathology, a session on muscle pathology is included.

During routine pathology work, muscle biopsies are channeled to the subspecialty pathologists for reporting. When interesting or diagnostically-typical cases are encountered, they are shared with trainees and interested colleagues.

Conclusions: Through the above teaching opportunities, trainees acquire a working knowledge of muscle pathology. From informal feedback, they appear to particularly like the casebased quiz teaching format. Many feel the discipline is relevant to their overall training in histopathology while some, if given the opportunity, may consider developing subspecialty interest in muscle pathology, auguring well for the future of the discipline.

\section{PATHOLOGY OF SURGICALLY EXCISED PRIMARY CARDIAC TUMORS: A REVIEW OF 39 YEAR SINGLE INSTITUTION EXPERIENCE}

\section{Felipe S. Templo Jr}

Division of Laboratory Medicine, Philippine Heart Center, Quezon City, Philippines

Background: Primary cardiac tumors are uncommon clinical entities, and thus, there are relatively limited descriptions in the published literature.
Aims: Most of these tumors have nonspecific presentations and thus, a clinico-pathologic review may provide additional information of potentially practical value for relevant clinical diagnosis and future translational researches.

Method: The surgical pathology files spanning a 39-year period (1975-2014) were reviewed for all surgically excised cardiac tumors. There were 353 of 357 (98.9\%) cases that were clinically established as primary.

Results: Of 353 primary cardiac tumors, 327 (92.6\%) were classified as benign, while $26(7.4 \%)$ were classified as malignant. There was female sex predilection for both benign and malignant tumors with a male to female ratio of $1: 2.8$. Of the benign tumors, the 5 most common histologic types were myxoma (95.1\%), fibroma (1.2\%), rhabdomyoma (1.2\%), hemangioma $(0.6 \%)$, and leiomyoma $(0.6 \%)$. The locations in decreasing frequency were left atrium, right atrium, left ventricle, and right ventricle. Tumor sizes ranged from 0.4 to $8.7 \mathrm{~cm}$. The average age of patient was 51.4 years. Among the malignant tumors, the 5 most common histologic types were myofibroblastic sarcoma (15.4\%), undifferentiated sarcoma (15.4\%), angiosarcoma (11.5\%), malignant fibrous histiocytoma $(11.5 \%)$ and rhabdomyosarcoma $(11.5 \%)$. Locations in decreasing frequency were left atrium, right atrium, right ventricle, ventricle and atrium, biventricular, and biatrial. Tumor sizes ranged from 0.8 to $10.6 \mathrm{~cm}$. Average age of patients was 39.8 years.

Conclusions: In this series, primary cardiac tumors generally have significant female sex predilection. Primary malignant cardiac tumors however occurred in a relatively younger age group than their benign counterparts.

\section{GENOMIC AND IMMUNOHISTOCHEMICAL PROFILES OF ENTEROPATHY-ASSOCIATED T-CELL LYMPHOMA IN JAPAN}

$\underline{\text { Sakura Tomita }^{1}}$, Yara Y. Kikuti ${ }^{1}$, Joaquim Carreras Esteban ${ }^{1}$, Katsuyoshi Takata $^{2}$, Tadashi Yoshino ${ }^{2}$, Sílvia Beà ${ }^{3}$, Elias Campo ${ }^{3}$, Naoya Nakamura

${ }^{1}$ Department of Pathology, Tokai University, School of Medicine, Isehara, ${ }^{2}$ Department of Pathology, Dentistry and Pharmaceutical Sciences, Okayama University Graduate School of Medicine, Okayama, Japan; and ${ }^{3}$ Hematopathology Unit, Hospital Clinic Barcelona, Institut d'Investigacions Biomediques August Pi i Sunyer (IDIBAPS), University of Barcelona, Barcelona, Spain

Background: Enteropathy-associated T-cell lymphoma (EATL) is a rare primary T-cell lymphoma of the digestive tract. EATL is classified as either Type I, which is frequently associated with and though to arise from celiac disease and is primarily observed in Northern Europe, and Type II, which occurs de novo and is distributed all over the world with predominance in Asia. Its pathogenesis in Asia is unknown.

Aims: We aimed to clarify the histological and genomic profiles of EATL in Japan in homogeneous series of 20 cases.

Methods: The cases were characterized by immunohistochemistry, high resolution oligonucleotide microarray, and fluorescence in situ hybridization (FISH) at 5 different loci: 1q21.3 (CKS1B), 6q16.3 (HACE1), 7p22.3 (MAFK), 9q33.3 (PPP6C) and 9q34.3 (ASS1, CARD9) using FFPE sections.

Results: The histological appearance ranged from medium- to large-sized cells in 13 cases (65\%), small-to-medium-sized cells 